\title{
Thoracolumbar Junction Syndrome Accompanying Renal Artery Stenosis: A Case Report
}

Kyudong Noh, $\mathrm{MD}^{1}$, Jong Burm Jung, $\mathrm{MD}^{2}$, Jeong Won Seong, $\mathrm{MD}^{3}$, Doh-Eui Kim, $\mathrm{MD}^{4}$, Dongrak Kwon, $\mathrm{MD}, \mathrm{PhD}^{5}$, Yuntae Kim, $\mathrm{MD}^{2}$

\begin{abstract}
${ }^{1}$ Department of Physical Medicine and Rehabilitation, Soonchunhyang University Bucheon Hospital, Soonchunhyang University College of Medicine, Bucheon; ${ }^{2}$ Department of Physical Medicine and Rehabilitation, Soonchunhyang University Cheonan Hospital, Soonchunhyang University College of Medicine, Cheonan; ${ }^{3}$ Department of Family Medicine, Sarang Clinic, Jinju; ${ }^{4}$ Department of Emergency Medicine, Soonchunhyang University Cheonan Hospital, Soonchunhyang University College of Medicine, Cheonan; ${ }^{5}$ Department of Rehabilitation Medicine, Catholic University of Daegu School of Medicine, Daegu, Korea
\end{abstract}

Flank pain is a common reason for visits to the emergency room. The most common reason warranting hospital visits are urology-related problems. However, there are many other causes, such as musculoskeletal lesions, that difficult to achieve a correct diagnosis. Here, we describe a rare case of flank pain caused by thoracolumbar junction syndrome, accompanying renal artery stenosis. A 54-year-old male with hypertension presented with severe left flank pain for 1 week. Initially, he was diagnosed with left renal artery stenosis by computed tomography and decreased renal function on renal scan (Tc-99m DTPA). Although a stent was inserted into the left renal artery, flank pain persisted with only minor improvement. Through detailed physical examination, he was finally diagnosed with thoracolumbar junction syndrome. After three injections in the left deep paravertebral muscles at the T10-T12 levels, flank pain completely ceased. Clinicians must consider thoracolumbar junction syndrome, when treating patients with flank pain.

Keywords Flank pain, Thoracolumbar junction syndrome, Renal artery stenosis

\section{INTRODUCTION}

Flank pain is commonly the result of one of three con- ditions, urinary tract infection, kidney stone, or musculoskeletal disorders [1]. Intraurinary causes of flank pain usually include stimulation of the visceral afferent nerve

Received June 24, 2019; Accepted July 17, 2019

Corresponding author: Yuntae Kim

Department of Physical Medicine and Rehabilitation, Soonchunhyang University Cheonan Hospital, Soonchunhyang University College of Medicine, 31 Suncheonhyang 6-gil, Dongnam-gu, Cheonan 31151, Korea. Tel: +82-41-570-2220, Fax: +82-41-592-3810, E-mail: simon108@schmc.ac.kr ORCID: Kyudong Noh (http://orcid.org/0000-0002-7207-6317); Jong Burm Jung (http://orcid.org/0000-0002-8343-2698); Jeong Won Seong (http:// orcid.org/0000-0003-3740-1732); Doh-Eui Kim (http://orcid.org/0000-0001-5499-6315); Dongrak Kwon (http://orcid.org/0000-0003-4728-930X); Yuntae Kim (http://orcid.org/0000-0003-4063-4692).

(ㄷ) This is an open-access article distributed under the terms of the Creative Commons Attribution Non-Commercial License (http://creativecommons.org/ licenses/by-nc/4.0) which permits unrestricted noncommercial use, distribution, and reproduction in any medium, provided the original work is properly cited. Copyright ( 2020 by Korean Academy of Rehabilitation Medicine 
upon distension of the ureter or renal capsule [2]. Abnormal radiologic findings for the genitourinary system play a critical role in diagnosing the problem. However, similar pain can be caused by extraurinary abnormalities, and it is difficult to distinguish it from the pain originating from the visceral structure. Physical examination plays a crucial role in identifying the location and characteristics of the pain.

Thoracolumbar junction (TLJ) syndrome is often characterized by pain around the iliac crest in the inguinal or lateral thigh area. This may result from dysfunction in the T10-L2 vertebral segments of the TLJ [3]. The vertebral segments responsible for visceral pain in the kidney are almost identical to those involved in TLJ syndrome. The clinical features of the two diseases may be similar $[4,5]$.

We describe a patient with flank pain around the iliac crest, initially considered to be caused by renal artery stenosis. Although the anatomical problems were resolved by angioplasty, the pain persisted and eventually improved by treating TLJ syndrome.

\section{CASE REPORT}

A 54-year-old male visited the urology outpatient clinic with left flank pain experienced for 1 week. He had no history of urinary stones and a 5-year history of hypertension with medication. He complained of sharp pain in the left flank and iliac crest areas (Fig. 1). The pain was

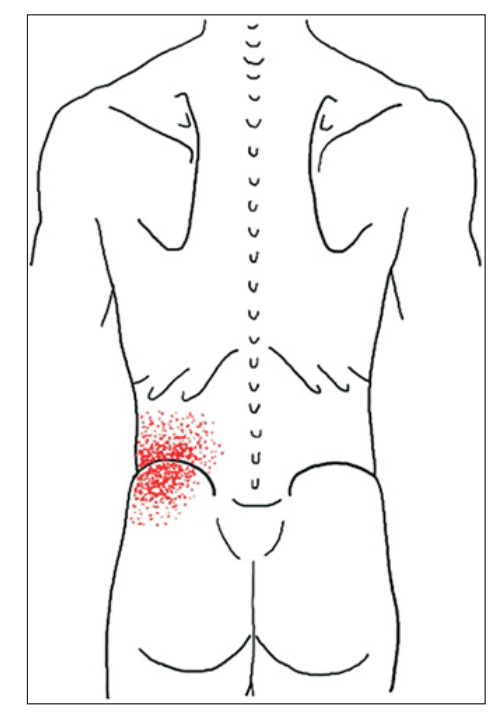

Fig. 1. Pattern of left flank pain from the thoracolumbar junction syndrome. rated at 10/10 on the Numeric Rating Scale (NRS). Initially, he visited a local clinic and was administered medication for pain control. However, he was referred to our hospital as there was no significant improvement in his symptoms. To evaluate the potential intraurinary causes, he was admitted to the department of urology. Several tests, including routine urine analysis, blood tests, and $\mathrm{X}$-ray were performed. Although there was no evidence of common intraurinary lesions, such as a urinary stones or urinary tract infection, he constantly complained of intense pain. He underwent abdominopelvic computed tomography (APCT), which indicated severe stenosis in the proximal portion of the left renal artery (Fig. 2). There were no abnormal findings in the spinal discs, facet joints and paraspinal muscles in APCT. Renal scan (Tc-99m DTPA [diethylenetriamine-pentaacetic acid]) was performed to evaluate his kidney function. It revealed that the glomerular filtration rate of left kidney was decreased (Fig. 3). The patient underwent percutaneous transluminal angioplasty, wherein a stent was inserted into the left renal artery. Pain improved by $5 / 10$ on the NRS, after intervention. Two days later he was discharged home.

However, the day after he was discharged, he returned to the emergency room with severely aggravated pain of $8 / 10$ on the NRS and was readmitted to the cardiology department. Flank pain worsened again despite stenting having been performed on the last admission, which was considered the cause of pain. The physician considered a

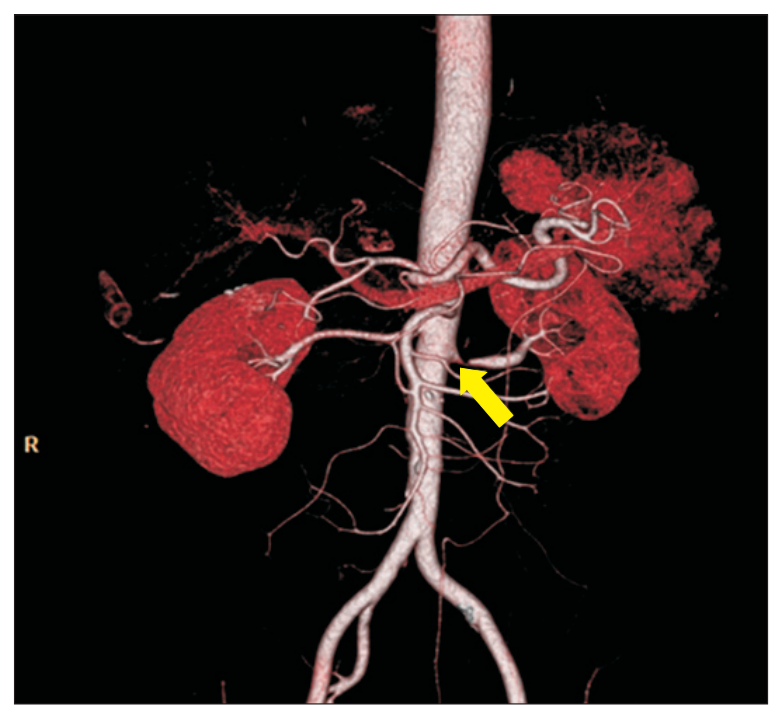

Fig. 2. Abdomen renal computed tomography angiography shows focal severe stenosis in the left proximal renal artery (arrow). 

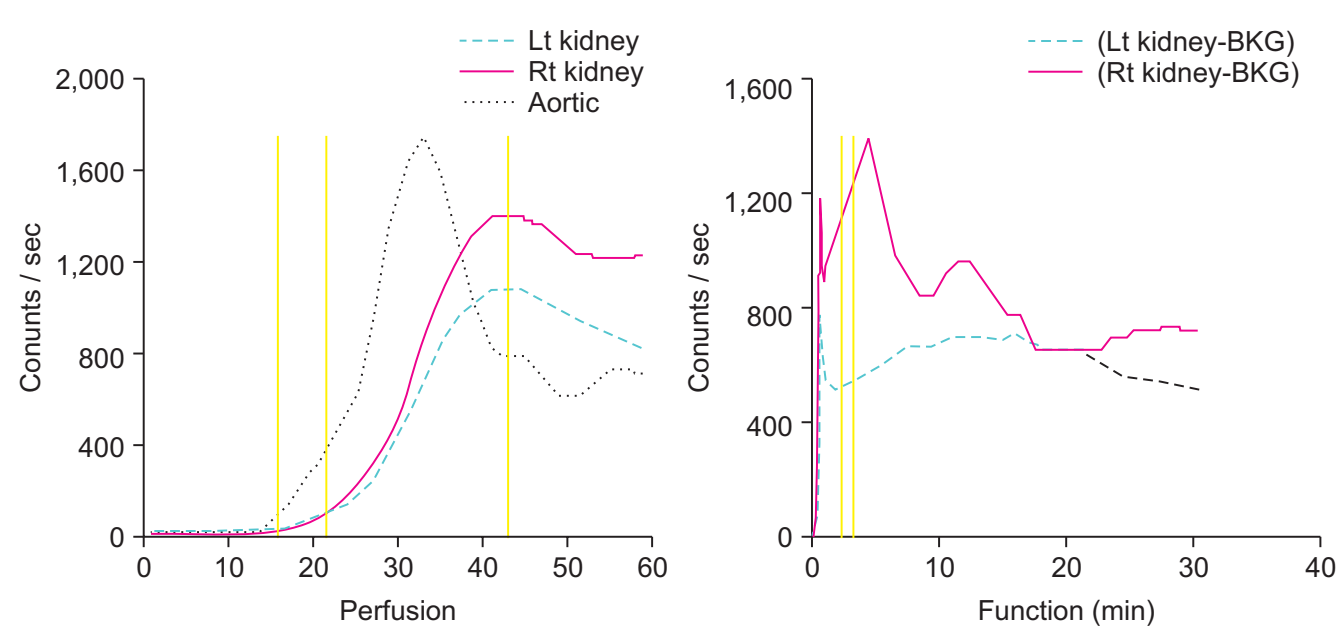

\begin{tabular}{|l|r|r|}
\hline \multicolumn{1}{|c|}{ Kidney } & Left & Right \\
\hline Kidney area $\left(\mathrm{cm}^{2}\right)$ & 52.06 & 69.41 \\
\hline Kidney depth (cm) & 6.27 & 6.31 \\
\hline Perfusion\% (Int) & 44.15 & 55.85 \\
\hline Perfusion\% (Slo) & 43.12 & 56.88 \\
\hline Uptake\% (Int) & 30.52 & 69.48 \\
\hline GFR & 25.30 & 57.61 \\
\hline Time to peak & 13.14 & 4.14 \\
\hline Peak to 1/2 peak & 41.72 & 13.00 \\
\hline
\end{tabular}

Fig. 3. Tc-99m DTPA diuretic renal scan demonstrated decreased glomerular filtration rate (GFR) in the left kidney.

musculoskeletal disorder.

A consultation with the rehabilitation department was conducted. During the physical examinations, tenderness was observed at each level of the left deep paravertebral muscles (DPVM) at the T10-T12 vertebrae. Local hyperalgesia on the skin over the left flank and iliac crest was identified when a fold of skin and subcutaneous tissue was pinched and rolled. Based on these findings, flank pain was thought to be the result of TLJ syndrome.

To correct the dysfunction of the TLJ segment, injections targeting the multifidus were administered, which plays the biggest role in the DPVM. Each needle was placed 1-1.5 cm lateral to the spinous process at the depth of the lamina. After touching the lamia, the physician pulled the needle approximately $5 \mathrm{~mm}$ backward and injected $3.5 \mathrm{~mL}$ of $0.25 \%$ lidocaine at each level. One area was injected per muscle and each of 3 levels were injected. There was no risk of blood vessel or nerve damage as the injection was completed after contact with the lamina bone. The average injection depth was approximately $3.5 \mathrm{~cm}$. The next day the patient reported significant pain relief and a decreased pain score from 8 to 3 on the NRS. A second injection had the same way. Following the second treatment, the patient was discharged home. When he visited the outpatient clinic 9 days after the second injection, the flank pain had resolved almost completely. As there was remaining tenderness in the left T10-T12 DPVM, an additional treatment was performed at these levels. Five days after the third injection, flank pain was completely resolved, and no recurrence was detected at the regular checkup at the cardiology depart- ment after several months.

Patient has provided informed consent for publication of the case.

\section{DISCUSSION}

We report a 54-year-old male who experienced acute flank pain accompanied by renal artery stenosis and TLJ syndrome. Clinically, it is difficult to determine the cause of flank pain for the following reasons.

Afferent impulses from the kidneys are primarily transmitted through the preganglionic sympathetic nerves to the spinal cord at the T10-L1 levels [6]. Renal ischemia, such as renal infarction and renal artery stenosis, can induce pain via the afferent nerve pathway to the spinal cord $[1,5]$. Additionally, there are many trunk muscles, which can be a cause of flank pain. The cutaneous branch of the T10-L1 levels can also be associated with flank pain. The lateral cutaneous branch from the dorsal ramus of the T7-T12 levels conveys the sensation of the area from the subscapular to the iliac crest. The nerves of the T11-L2 levels are a sensory fiber to the area under the iliac crest [7]. In this case, renal ischemia occurring from renal artery stenosis was initially thought to be the cause of the flank pain.

Above all, the medical causes related to the intraurinary condition must be diagnosed for proper treatment to take place. APCT is a reliable test to diagnosis these problems. Other musculoskeletal causes can be detected by computed tomography or magnetic resonance imaging. However, physical examination is more important as 
symptoms and radiological results are not always consistent.

Maigne [3] described the TLJ syndrome as a condition, typically, with pain on the iliac crest, and tenderness on palpation of the TLJ. As mentioned above, lateral branches of dorsal ramus at the T7-L2 levels have cutaneous innervation after passing through the DPVM. These nerves can be functionally entrapped to the point of piercing the DPVM. Seong [8] mentioned that excessive tension and functional shortening of the DPVM can increase pressure of the vertebral foramen, which can result in excitation of the ventral and dorsal ramus of the spinal nerve. If the vasa nervorum of the spinal nerve is compressed, focal ischemia can be induced. Adenosine triphosphate (ATP) production can decrease, causing failure of the $\mathrm{Na}+/ \mathrm{K}+$ ATPase pump and elevation of the extra-membrane $\mathrm{K}+$ concentration. This may increase the resting membrane potential or reduce the threshold of nerve cells, leading to membrane hyperexcitability [9]. Hyperexcited nerve fiber can produce abnormal excitation signals and cause various types of pain. In this case, flank pain was the result of entrapment of the dorsal ramus of the spinal nerve at the T10-T12 levels.

For diagnosis of TLJ syndrome, it is crucial to examine tender points by palpation of the DPVM in the thoracolumbar spine. Additionally, the skin and subcutaneous tissue of the flank and lower lumbar must be examined with the pinch-roll test. This test is accompanied by hyperalgesia at the involved dermatomes. Diagnosis of TLJ syndrome is confirmed by symptoms relieved after DPVM injection.

Conventional treatments of TLJ syndrome include manipulation targeted at restoring motion in the hypomobile segments and injection therapy at the involved segments [3]. A functional restoration program and several exercises, such as pelvic tilt exercises and hip bearing exercises, may also be considered [10].

In conclusion, TLJ syndrome is responsible in a considerable number of cases in patients with flank pain. In patients with flank pain whose internal organ problems have been dealt with, injecting saline or diluted lidocaine surrounding the painful DPVM and around the T12-L1 segments can easily relieve flank pain. A physical examination such as the pinch-roll test for somatic sensory nerve entrapment is crucial for accurate diagnosis. Releasing entrapment of the involved spinal segments is a fundamental treatment and can be conducted with simple injection therapy.

\section{CONFLICT OF INTEREST}

No potential conflict of interest relevant to this article was reported.

\section{ACKNOWLEDGMENTS}

This work was supported by the Soonchunhyang University Research Fund and the TongSa (Association of Pain and Function Analysis) Research Fund.

\section{AUTHOR CONTRIBUTION}

Conceptualization: Noh KD, Kim YT, Seong JW. Writing - original draft: Noh KD. Writing - review \& editing: Kim YT, Jung JB, Kwon DR, Kim DE.

\section{REFERENCES}

1. Bueschen AJ. Flank pain. In: Walker HK, Hall WD, Hurst JW, editors. Clinical methods: the history, physical, and laboratory examinations. 3rd ed. Boston: Butterworths; 1990. p. 845-6.

2. Smith RC, Levine J, Dalrymple NC, Barish M, Rosenfield AT. Acute flank pain: a modern approach to diagnosis and management. Semin Ultrasound CT MR 1999;20:108-35.

3. Maigne R. Thoracolumbar junction syndrome. In: Diagnosis and treatment of pain of vertebral origin. 2nd ed. Boca Raton, FL: CRC Press; 2006. p. 383-8.

4. Singh RR, McArdle ZM, Iudica M, Easton LK, Booth LC, May CN, et al. Sustained Decrease in blood pressure and reduced anatomical and functional reinnervation of renal nerves in hypertensive sheep 30 months after catheter-based renal denervation. Hypertension 2019;73:718-27.

5. Kirkpatrick JJ, Leslie SW. Anatomy, abdomen and pelvis, kidney nerves [Internet]. Treasure Island, FL: StatPearls Publishing; 2019 [cited 2020 Jan 15]. Available from: https://www.ncbi.nlm.nih.gov/ pubmed/29083631.

6. Shokeir AA. Renal colic: new concepts related to pathophysiology, diagnosis and treatment. Curr Opin 
Urol 2002;12:263-9.

7. Maigne R. Low back pain of thoracolumbar origin (T11-T12-L1). In: Diagnosis and treatment of pain of vertebral origin. 2nd ed. Boca Raton, FL: CRC Press; 2006. p. 289-98.

8. Seong JW. Principle and insight into pain. Paju, Korea: Koonja Press; 2012. p. 844-55.

9. Han SE, Lin CS, Boland RA, Kiernan MC. Nerve com- pression, membrane excitability, and symptoms of carpal tunnel syndrome. Muscle Nerve 2011;44:402-9. 10. Alptekin K, Ornek NI, Aydın T, Alkan M, Toprak M, A Balcı L, et al. Effectiveness of exercise and local steroid injections for the thoracolumbar junction syndrome (The Maigne's syndrome) treatment. Open Orthop J 2017;11:467-77. 